\title{
Stabilisation of a Flexible Spacecraft Subject to External Disturbance and Uncertainties
}

\author{
Yun Fu $\mathbb{D},{ }^{1}$ Yu Liu, ${ }^{2}$ Lingyan $\mathrm{Hu}^{1}$ and Lingxi Peng $\mathbb{D}^{3}$ \\ ${ }^{1}$ School of Information Engineering, Nanchang University, Nanchang 330031, China \\ ${ }^{2}$ School of Automation Science and Engineering, South China University of Technology, Guangzhou 510640, China \\ ${ }^{3}$ School of Mechanical and Electrical Engineering, Guangzhou University, Guangzhou 510006, China
}

Correspondence should be addressed to Lingxi Peng; scu.peng@gmail.com

Received 1 May 2020; Accepted 18 June 2020; Published 23 July 2020

Academic Editor: Shuping He

Copyright (c) 2020 Yun Fu et al. This is an open access article distributed under the Creative Commons Attribution License, which permits unrestricted use, distribution, and reproduction in any medium, provided the original work is properly cited.

\begin{abstract}
This paper addresses the problems of vibration reduction and attitude tracking for a flexible spacecraft subject to external disturbances and uncertainties. Based on Hamilton's principle, flexible spacecraft is modelled by a coupled nonlinear partial differential equation with ordinary differential equations. Adaptive boundary control scheme is adopted to stabilize the vibration displacement of flexible appendage into a small neighbourhood of original position and simultaneously maintain attitude angle within the desired angle region. Two disturbance adaptive laws are constructed to attenuate the effect of unknown external disturbances. The well posedness of the controlled system is proven by using the semigroup theory. The proposed adaptive boundary control scheme can guarantee the uniform boundedness of the closed-loop system. Numerical simulation results illustrate the effectiveness of the proposed control scheme.
\end{abstract}

\section{Introduction}

Spacecraft with flexible appendage is playing a key role in the development of the communication industry and remote sensing. To satisfy the space mission requirements, attitude maneuver is the basic operation of spacecraft. In the complex space environment, flexible appendage may vibrate under the effects of external disturbances and attitude maneuver. Flexible appendage generally possesses characteristics of light weight, low damping, and limited energy. These flexible characteristics make the vibration reduction slow down. However, the continuous vibration may reduce the operating efficiency of flexible spacecraft and even result in the destruction of flexible appendage. Hence, it is necessary to design a powerful and efficient controller to reduce the vibration of flexible appendage and simultaneously track the desired attitude.

In recent years, many research studies related to the modeling, vibration suppression, and attitude control of flexible spacecraft have been published. For the sake of control design, the models of flexible spacecraft are discretized in most of these works. Examples of these control schemes include fault tolerant control [1], optimal control [2], and positive position feedback control [3]. In [4], a variable structure control based on pulse-width pulse-frequency technology is designed to suppress the vibration of a flexible spacecraft with parameter uncertainties and input nonlinearity. In [5], using the distributed piezoelectric actuator technology, the authors propose a momentum exchange feedback control to stabilize flexible spacecraft. In [6], $H_{\infty}$ state feedback control is proposed for a flexible spacecraft in the presence of the multiobjective design requirements. The above control schemes are developed based on the discretization ordinary differential equation (ODE) models. However, model discretization may make the model inaccurate and bring spillover instability in control system although the ODE model provides the convenience in design control and appears brief in form [7,8].

Compared with the control schemes designed from the ODE model [9-11], boundary control and distributed control are derived from the original partial differential equation (PDE) model, which can control all system modes 
and eliminate the drawback of spillover instability. They have been widely used in the vibration reduction of flexible mechanical systems [12-14]. Synchronization control [15], backstepping boundary control [16], and cooperative control [17] are also effective for the distributed parameter system. In [18], the distributed fuzzy controller is adopted for a nonlinear distributed parameter system. It must be noted that distributed control is effective for the distributed parameter system. In [19], the authors design a mixed fuzzy/ boundary control to ensure the practical stability of a nonlinear beam system. However, distributed control is more difficult to be implemented than boundary control in practice since it requires many distributed actuators and senors. Hence, boundary control is regarded as a more practical control scheme. In $[20,21]$, the authors design the restricted boundary controls to stabilize the flexible aerial refueling hose systems in the presence of varying length and speed. In [22], adaptive boundary control is designed to suppress the vibration of the flexible axially moving belt system with high acceleration/deceleration. Boundary barrier-based control is developed to regulate the elastic deformation of a flexible crane system with output constraint in [23]. In [24, 25], output feedback controls are developed to realize the target of vibration reduction for nonlinear flexible strings. For the nonuniform flexible wind turbine tower system, boundary control strategy is developed to reduce vibration via the generator electric torque in [26]. Although the great progress has been acquired for the boundary control design of flexible mechanical systems, the study of adaptive boundary control design for a flexible spacecraft system subject to external disturbances and parameter uncertainties is few. These results motivate us to design an adaptive boundary control scheme for the flexible spacecraft system.

External disturbance exists widely in practical engineering application. Many disturbance rejection technologies have been developed [27-29]. In [30], the signum function is considered to attenuate the impact of external disturbance, where the exact value of the upper bound of external disturbance is known. However, the signum function may bring the chattering in control input and the accurate information of external disturbance is hard to determine in practice. In [31], a common disturbance observer is developed to compensate for the effect of the bounded disturbance with the bounded rate, where the system structure physical parameters are certain. However, the robustness of the disturbance observers proposed in these papers is weak and the structure physical parameters of flexible spacecraft are uncertain. Hence, handling the effect of external disturbances in control design for a flexible spacecraft with uncertain parameters is still challenging. Mathematically, the proof of well posedness is one of the most important aspects of the stability analysis of the distributed parameter system. In [32], using the spectral analysis based on Lyapunov approach, the exponential stability of the Euler-Bernoulli beam system and the behavior of the solution of the closed-loop system are investigated. In [33-35], employing Gelerkin's approximation method, the well posedness of the flexible beam system with the proposed feedback boundary control is discussed. To avoid the complicated and tedious functional calculations, the semigroup theory is used to prove the well posedness of the closed-loop system in this paper.

In this paper, we investigate the vibration reduction and attitude control of a flexible spacecraft with parameter uncertainties. The accurate dynamic model of flexible spacecraft is given by a set of coupled partial differential equation with ordinary differential equations. Two adaptive boundary control laws are designed to ensure the uniformly bounded stability of the closed-loop system. It should be noted that the proposed control scheme is derived from the original infinite dimensional dynamic model without any discretization, which can avoid spillover instability. Combining robust control strategy with two disturbance adaptive laws, the effect of external disturbances is attenuated exponentially in the negative feedback loop. The proposed disturbance rejection method can avoid chattering phenomenon and improve robust of the designed control scheme. The well posedness and uniform boundedness of the closed-loop system are proven under the semigroup theory and Lyapunov stability theory.

The paper is organized as follows. The model of flexible spacecraft is derived in Section 2. Adaptive boundary control scheme with disturbance adaptive laws is designed in Section 3. In Section 4, the well posedness and stability of the closedloop system are discussed. The results of numerical simulations are given in Section 5. Finally, this paper is concluded in Section 6.

\section{Dynamics Analysis}

2.1. Dynamic Model. Figure 1 shows a typical model of flexible spacecraft. The model consists of a rigid hub with the radius $r$, which represents the spacecraft body, and an uniform flexible cantilever beam with the tip mass $m$, which represents the flexible appendage, such as solar array or any other flexible structure. $O X Y$ and $o x y$ are defined as the inertial frame and the frame fixed on the hub, respectively. Denote $w(x, t)$ as the elastic deflection at point $x$ and time $t$ with respect to the oxy frame. Define $\theta(t)$ as the attitude angle. $\theta_{d}$ is defined as the desired attitude. The tracking error is defined as $\theta_{e}(t)=\theta(t)-\theta_{d} \cdot d_{1}(t)$ and $d_{2}(t)$ are the unknown boundary disturbances. $u_{1}(t)$ and $u_{2}(t)$ are the control inputs. The structure physical parameters of the flexible spacecraft system are listed as follows: EI is the bending stiffness of the flexible appendage, $c$ is the coefficient of viscous damping, $l$ is the length of the flexible appendage, $T$ is the tension, $\rho$ is the uniform mass per unit length, and $I_{h}$ denotes the hub inertia.

For the sake of convenience in writing, the following notations are used throughout this paper: $(\cdot)=$ $(\cdot)(x, t),(\cdot)=\partial(\cdot) / \partial t,(\cdot)=\partial^{2}(\cdot) / \partial t^{2},(\cdot)^{\prime}=\partial(\cdot) / \partial x,(\cdot)^{\prime \prime}=$ $\partial^{2}(\cdot) / \partial x^{2},(\cdot)^{\prime \prime \prime}=\partial^{3}(\cdot) / \partial x^{3},(\cdot)^{\prime \prime \prime \prime}=\partial^{4}(\cdot) / \partial x^{4},(\cdot)^{(n)}=\partial^{n}(\cdot) /$ $\partial x^{n}$.

To obtain the PDE model of the flexible spacecraft system, the kinetic energy, potential energy, and virtual work can be expressed as follows: 


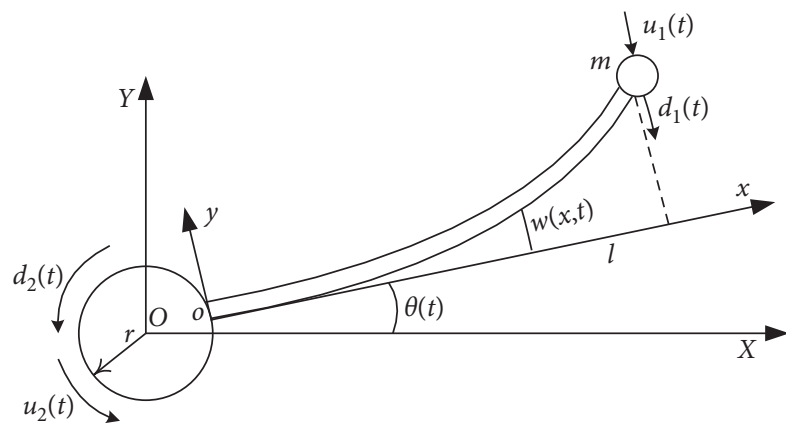

Figure 1: A typical flexible spacecraft system.

$$
\begin{aligned}
E_{k}(t)= & \frac{1}{2} \rho \int_{0}^{l}[\dot{w}+(r+x) \dot{\theta}(t)]^{2} \mathrm{~d} x+\frac{1}{2} I_{h} \dot{\theta}^{2}(t) \\
& +\frac{1}{2} m[\dot{w}(l, t)+(r+l) \dot{\theta}(t)]^{2}, \\
E_{p}(t)= & \frac{1}{2} T \int_{0}^{l}\left(w^{\prime}\right)^{2} \mathrm{~d} x+\frac{1}{2} \mathrm{EI} \int_{0}^{l}\left(w^{\prime \prime}\right)^{2} \mathrm{~d} x, \\
\delta W(t)= & -c \int_{0}^{l}[\dot{w}+(r+x) \dot{\theta}(t)] \delta[w+(r+x) \theta(t)] \mathrm{d} x \\
& +\left[u_{1}(t)+d_{1}(t)\right] \delta[w(l, t)+(r+l) \theta(t)] \\
& +\left[u_{2}(t)+d_{2}(t)\right] \delta \theta(t) .
\end{aligned}
$$

Using Hamilton's principle $\int_{t_{1}}^{t_{2}}\left[\delta E_{k}(t)-\delta E_{p}(t)+\right.$ $\delta W(t)] \mathrm{d} t=0$, variational principle, and integration by parts, we can obtain the governing equations of the flexible spacecraft system as

$$
\begin{aligned}
& \rho \ddot{w}+\mathrm{EI} w^{\prime \prime \prime \prime}-T w^{\prime \prime}+c \dot{w}=-(r+x)[\rho \ddot{\theta}(t)+c \dot{\theta}(t)], \\
& I_{h} \ddot{\theta}(t)=\mathrm{EI} w^{\prime \prime \prime}(0, t)-r \mathrm{EI} w^{\prime \prime \prime}(0, t)+T w(l, t)+u_{2}(t) \\
& +d_{2}(t),
\end{aligned}
$$

and the boundary conditions as

$$
\begin{aligned}
& m \ddot{w}(l, t)=-m(r+l) \ddot{\theta}(t)+\mathrm{EI} w^{\prime \prime \prime}(l, t)-T w^{\prime}(l, t) \\
&+u_{1}(t)+d_{1}(t), \\
& w(0, t)=w^{\prime}(0, t)=w^{\prime \prime}(l, t)=0 .
\end{aligned}
$$

2.2. Preliminaries. The following lemmas and assumption are proposed for the convenience of control design and stability analysis.

Lemma 1. Let $w(x, t)$ be the continuously differentiable function with $w(0, t)=0$; then, the following inequality holds [36]:

$$
w^{2}(x, t) \leq l \int_{0}^{l}\left[w^{\prime}(x, t)\right]^{2} \mathrm{~d} x, \quad \forall t \in[0, \infty)
$$

Lemma 2. For bounded initial conditions, if there exists a $C^{1}$ continuous Lyapunov function $V(x)>0$ satisfying $a_{1}(\|x\|) \leq V(x) \leq a_{2}(\|x\|)$, such that $\dot{V}(x) \leq-b V(x)+c$, where $a_{1}, a_{2}: R^{n} \longrightarrow R$ are class $\mathscr{K}$ functions and $b, c>0$, then the solution $x(t)$ is uniformly bounded [37].

Assumption 1. For the unknown boundary disturbances $d_{1}(t)$ and $d_{2}(t)$, we assume that there exist two positive constants $\bar{d}_{1}$ and $\bar{d}_{2}$, such that $d_{1}(t) \leq \bar{d}_{1}$ and $d_{2}(t) \leq \bar{d}_{2}$, $\forall t \in[0, \infty)$. In practice, the energy of external disturbances $d_{1}(t)$ and $d_{2}(t)$ is finite. Thus, this assumption is reasonable.

\section{Control Design}

The control objectives of this paper are to reduce the vibration of flexible appendage and simultaneously trace the desired attitude. The block diagram given by Figure 2 describes the design procedure of the control strategy proposed in this paper for a flexible spacecraft system with external disturbances and parameter uncertainties. To stabilize the flexible spacecraft system described by (2)-(5), we design the following two adaptive boundary control laws:

$$
\left\{\begin{array}{c}
u_{1}(t)=-k_{0} u_{a}(t)-\widehat{m}\left[k_{1} \dot{w}^{\prime}(l, t)-k_{2} \dot{w}^{\prime \prime \prime}(l, t)\right] \\
+\widehat{T} w^{\prime}(l, t)-\widehat{E I} \dot{w}^{\prime \prime \prime}(l, t)+u_{d_{1}}(t), \\
u_{2}(t)=-k_{3} \theta_{e}(t)-k_{4} S(t)-\gamma_{1} \widehat{I}_{h} \dot{\theta}(t)+u_{d_{2}}(t),
\end{array}\right.
$$

where $\gamma_{1}, k_{0}, k_{1}, k_{2}, k_{3}, k_{4}>0, \widehat{m}, \widehat{T}, \widehat{E I}$, and $\widehat{I}_{h}$ are the system parameter estimates, and the auxiliary signals $u_{a}(t)$ and $S(t)$ are given as follows:

$$
\left\{\begin{array}{l}
u_{a}(t)=\dot{w}(l, t)+(r+l) \dot{\theta}(t)+k_{1} w^{\prime}(l, t)-k_{2} w^{\prime \prime \prime}(l, t), \\
S(t)=\gamma_{1} \theta_{e}(t)+\dot{\theta}(t),
\end{array}\right.
$$

where $u_{d_{1}}(t)$ and $u_{d_{2}}(t)$ are two new input signals and are proposed as follows:

$$
\left\{\begin{array}{l}
u_{d_{1}}(t)=-\frac{\widehat{d}_{1}^{2}(t)}{\widehat{d}_{1}(t)\left|u_{a}(t)\right|+\tau_{1}} u_{a}(t), \\
u_{d_{2}}(t)=-\frac{\widehat{d}_{2}^{2}(t)}{\widehat{d}_{2}(t)|S(t)|+\tau_{2}} S(t),
\end{array}\right.
$$

in which $\tau_{1}, \tau_{2}>0$ and $\widehat{d}_{1}(t)$ and $\widehat{d}_{2}(t)$ are the estimates of $\bar{d}_{1}$ and $\bar{d}_{2}$.

For a flexible spacecraft system with parameter uncertainties, we consider the following adaptive laws to estimate system parameters 




FIGURE 2: Design procedure of the adaptive boundary control scheme.

$$
\begin{cases}\dot{\widehat{m}}=-\eta_{1} \widehat{m}+u_{a}(t)\left[k_{1} \dot{w}^{\prime}(l, t)-k_{2} \dot{w}^{\prime \prime \prime}(l, t)\right], & \widetilde{m}=\widehat{m}-m, \\ \dot{\widehat{T}}=-\eta_{2} \widehat{T}-u_{a}(t) w^{\prime}(l, t), & \widetilde{T}=\widehat{T}-T, \\ \dot{\mathrm{EI}}=-\eta_{3} \widehat{\mathrm{EI}}+u_{a}(t) w^{\prime \prime \prime}(l, t), & \widetilde{\mathrm{EI}}=\widehat{\mathrm{EI}}-\mathrm{EI}, \\ \dot{\widehat{I}}_{h}=-\eta_{4} \widehat{I}_{h}+\gamma_{1} S(t) \dot{\theta}(t), & \widetilde{I}_{h}=\widehat{I}_{h}-I_{h},\end{cases}
$$

in which $\widetilde{m}, \widetilde{T}, \widetilde{E I}$, and $\widetilde{I}_{h}$ are the estimation errors of system parameters.

The following disturbance adaptive laws are constructed to handle unknown boundary disturbances:

$$
\begin{aligned}
& \left\{\begin{array}{l}
\dot{\hat{d}}_{1}(t)=-\sigma_{1} \hat{d}_{1}(t)+\left|u_{a}(t)\right|, \\
\tilde{d}_{1}(t)=\hat{d}_{1}(t)-\bar{d}_{1},
\end{array}\right. \\
& \left\{\begin{array}{l}
\dot{\vec{d}}_{2}(t)=-\sigma_{2} \hat{d}_{2}(t)+|S(t)|, \\
\tilde{d}_{2}(t)=\widehat{d}_{2}(t)-\bar{d}_{2},
\end{array}\right.
\end{aligned}
$$

where $\widetilde{d}_{1}(t)$ and $\widetilde{d}_{2}(t)$ are the estimation errors of $\bar{d}_{1}$ and $\bar{d}_{2}$.

Remark 1. All signals in the proposed adaptive boundary control scheme (7) can be measured by sensors or computed by backward difference algorithm. $w(l, t), w^{\prime}(l, t), w^{\prime \prime \prime}(l, t)$, $\theta(t)$, and $\dot{\theta}(t)$ can be directly measured by laser displacement sensor, inclinometer, shear force sensor, rotary encoder, and tachometer, respectively. $\dot{w}^{\prime}(l, t)$ and $\dot{w}^{\prime \prime \prime}(l, t)$ can be computed by using backward difference algorithm based on measured values.

Remark 2. In this paper, different from the traditional control schemes designed from the ODE model, the adaptive boundary control scheme given by (7) is designed directly based on the original PDE model. Therefore, the drawback of spillover instability can be avoided and all system modes can be controlled. Moreover, the proposed boundary scheme (7) is an easier implemented control scheme than distributed control since it only requires the actuators and sensors at the system boundaries.

Remark 3. In practice, the exact values of boundary disturbances and the system structure physical parameters of flexible spacecraft are uncertain. To handle the unknown boundary disturbances, two disturbance adaptive laws (11) and (12) are developed, where we only require to ensure the existence of $\bar{d}_{1}$ and $\bar{d}_{2}$ and the chattering can be avoided. Thus, the proposed adaptive boundary control scheme (7) has better robustness than the common disturbance rejection technologies such as the signum function and disturbance observers.

\section{Stability Analysis}

4.1. Well-Posed Problem. In this part, the well posedness of the closed-loop system given by (2)-(5) is proven by employing the semigroup theory. The Hilbert space is introduced as the functional space. And then the closed-loop system can be rewritten to a first-order evolution equation. This result means that many results of the ordinary differential equation system can be applied in the proposed flexible spacecraft system.

For analyzing the well posedness, we introduce the following new variables:

$$
\left\{\begin{array}{l}
\widehat{\Phi}=[\widehat{m}, \widehat{T}, \widehat{E I}]^{T} \\
\widetilde{\Phi}=[\widetilde{m}, \widetilde{T}, \widetilde{E I}]^{T} \\
\Psi=\left[k_{1} \dot{w}^{\prime}(l, t)-k_{2} \dot{w}^{\prime \prime \prime}(l, t),-w^{\prime}(l, t), w^{\prime \prime \prime}(l, t)\right]^{T} \\
z(x, t)=w(x, t)+(r+x) \theta(t) \\
w_{e}(x, t)=w(x, t)+(r+x) \theta_{e}(t)
\end{array}\right.
$$

The flexible spacecraft system described by (2)-(5) can be transformed as the following closed-loop system: 


$$
\left\{\begin{array}{l}
\rho \ddot{z}+E I z^{\prime \prime \prime \prime}-T z^{\prime \prime}+c \dot{z}=0, \\
I_{h} \dot{S}(t)=-k_{4} S(t)-\gamma_{1} \widetilde{I}_{h} \dot{\theta}(t)-k_{3} \theta_{e}(t)+E I z^{\prime \prime}(0, t) \\
\quad-r E I z^{\prime \prime \prime}(0, t) \\
+d_{2}(t)+u_{d_{2}}(t)+T[z(l, t)-(r+l) \theta(t)] \\
m \dot{u}_{a}(t)=-k_{0} u_{a}(t)+d_{1}(t)+u_{d_{1}}(t)-\Psi^{T} \widetilde{\Phi} \\
\theta(t)=z^{\prime}(0, t)=\frac{1}{r} z(0, t) \\
z^{\prime \prime}(l, t)=0 .
\end{array}\right.
$$

Define the state space as follows:

$$
H \triangleq H_{L}^{2}(0 . l) \times H_{L}^{2}(0, l) \times L^{2}(0, l) \times R^{2},
$$

where the space $L^{2}$ and $H_{L}^{2}$ are defined as

$$
\begin{aligned}
L^{2}(0, l)= & \left\{f:[0, l] \longrightarrow R \mid \int_{0}^{l} f^{2} \mathrm{~d} x<\infty\right\}, \\
H_{L}^{2}(0, l)= & \left\{f \in L^{2}(0, l) \mid \frac{\partial f}{\partial x}, \frac{\partial^{2} f}{\partial x^{2}} \in L_{2}, f(0)=r f^{\prime}(0),\right. \\
& \left.f^{\prime \prime}(l)=0\right\} .
\end{aligned}
$$

The inner product of $H$ is considered as

$$
\begin{aligned}
\left\langle Y_{1}, Y_{2}\right\rangle_{H}= & \frac{\rho}{2} \int_{0}^{l} \psi_{1} \psi_{2} \mathrm{~d} x+\frac{\mathrm{EI}}{2} \int_{0}^{l}\left(\omega_{1}^{\prime \prime}\right)\left(\omega_{2}^{\prime \prime}\right) \mathrm{d} x \\
& +\frac{T}{2} \int_{0}^{l}\left[\omega_{1}^{\prime}-\omega_{1}^{\prime}(0)\right]\left[\omega_{2}^{\prime}-\omega_{2}^{\prime}(0)\right] \mathrm{d} x+\frac{m}{2} u_{a_{1}} u_{a_{2}} \\
& +\frac{I_{h}}{2} S_{1} S_{2}+\frac{k_{3}}{2 \gamma_{1}^{2}}\left[S_{1}-\psi_{1}^{\prime}(0)\right]\left[S_{2}-\psi_{2}^{\prime}(0)\right] \\
& +\gamma_{1} \rho \int_{0}^{l}(r+x)\left[\left(\varphi_{1}^{\prime}+\psi_{1}\right)\left(\varphi_{2}^{\prime}+\psi_{2}\right)\right. \\
& \left.-\varphi_{1}^{\prime} \varphi_{2}^{\prime}-\psi_{1} \psi_{2}\right] \mathrm{d} x,
\end{aligned}
$$

where $Y_{i}=\left(\omega_{i}, \varphi_{i}, \psi_{i}, u_{a_{i}}, S_{i}\right)^{T} \in H, i=1,2$.

We construct a linear operator as

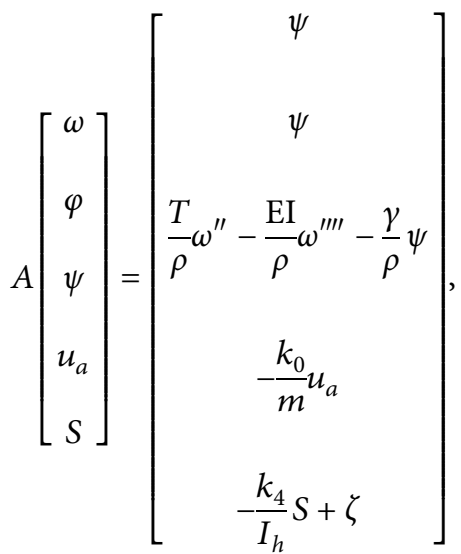

with its domain

$$
\begin{gathered}
D(A)=\left\{\left(\omega, \varphi, \psi, u_{a}, S\right)^{T} \in H \mid S=\gamma_{1} \varphi^{\prime}(0)+\frac{1}{r} \psi^{\prime}(0),\right. \\
\left.u_{a}=\psi(l)+k_{1}\left[\omega^{\prime}(l)-\omega^{\prime}(0)\right]-k_{2} \omega^{\prime \prime \prime}(l)\right\},
\end{gathered}
$$

where

$$
\begin{aligned}
\zeta= & -\frac{k_{3}}{I_{h}} \varphi^{\prime}(0)+\frac{\mathrm{EI}}{I_{h}} \omega^{\prime \prime}(0)-\frac{r \mathrm{EI}}{I_{h}} \omega^{\prime \prime \prime}(0) \\
& +\frac{T}{I_{h}}\left[\omega(l)-(r+l) \omega^{\prime}(0)\right] .
\end{aligned}
$$

Therefore, the closed-loop system (15) can be rewritten by the following evolutionary equation:

$$
\left\{\begin{array}{l}
\frac{\mathrm{d} Y(t)}{\mathrm{d} t}=A Y(t)+F(t), \\
Y(0)=Y_{0},
\end{array}\right.
$$

where $Y(t)=\left[z(\cdot, t), w_{e}(\cdot, t), \dot{z}(\cdot, t), u_{a}(t), S(t)\right]^{T}, Y_{0}$ is the system initial state, and $F(t)$ is given as follows:

$$
F(t)=\left[\begin{array}{c}
0 \\
0 \\
0 \\
\frac{1}{m}\left[d_{1}(t)+u_{d_{1}}(t)-\Psi^{T} \widetilde{\Phi}\right] \\
\frac{1}{I_{h}}\left[d_{2}(t)+u_{d_{2}}(t)-\frac{\gamma_{1} \widetilde{I}_{h}}{r} \psi^{\prime}(0, t)\right]
\end{array}\right] .
$$


For any $Y \in H$,

$$
\begin{aligned}
\langle Y, A Y\rangle_{H} \leq & -M\left[\int_{0}^{l} \psi^{2} \mathrm{~d} x+\int_{0}^{l}\left[\omega^{\prime}-\omega^{\prime}(0)\right]^{2} \mathrm{~d} x\right. \\
& \left.+\int_{0}^{l}\left(\omega^{\prime \prime}\right)^{2} \mathrm{~d} x+u_{a}^{2}+\int_{0}^{l} \varphi^{2} \mathrm{~d} x+S^{2}+\theta_{e}^{2}(t)\right] \leq 0,
\end{aligned}
$$

where $M$ is a positive constant. Then, the operator $A$ given by (18) is dissipative in $H$.

We claim that $A^{-1}$ is compact on $H$. For any $Q=\left(q_{1}, q_{2}, q_{3}, q_{4}, q_{5}\right)^{T} \in H$, consider the solvability of equation $A Y=Q, Y=\left(\omega, \varphi, \psi, u_{a}, S\right)^{T} \in D(A)$. From (18), we can obtain the following equation:

$$
\left\{\begin{array}{l}
\psi(x)=q_{1}(x), \\
\psi(x)=q_{2}(x), \\
\frac{T}{\rho} \omega^{\prime \prime}(x)-\frac{\mathrm{EI}}{\rho} \omega^{\prime \prime \prime \prime}(x)-\frac{\gamma}{\rho} \psi(x)=q_{3}(x), \\
-\frac{k_{0}}{m} u_{a}=q_{4}, \\
\frac{k_{4}}{I_{h}} S+\zeta=q_{5},
\end{array}\right.
$$

with corresponding boundary conditions

$$
\left\{\begin{array}{l}
\omega(0)=r \omega^{\prime}(0), \\
\omega^{\prime \prime}(l)=0, \\
S=\gamma_{1} \varphi^{\prime}(0)+\frac{1}{r} \psi^{\prime}(0), \\
u_{a}=\psi(l)+k_{1}\left[\omega^{\prime}(l)-\omega^{\prime}(0)\right]-k_{2} \omega^{\prime \prime \prime}(l) .
\end{array}\right.
$$

Solving (24) leads to

$$
\left\{\begin{array}{l}
\omega(x)=\frac{e^{-b x}}{\mathrm{EI}} \int_{0}^{x} e^{2 b g} \int_{0}^{g} e^{-b \varsigma} \int_{0}^{\varsigma} \int_{0}^{p}\left[\rho q_{3}(s)+\gamma q_{1}(s)\right] \\
\mathrm{d} s \mathrm{~d} p \mathrm{~d} \varsigma \mathrm{d} g+a_{1}+a_{2} x+a_{3} e^{-b x}+a_{4} e^{b x} \\
\varphi(x)=\omega(x)-(r+x) \theta_{d} \\
\psi(x)=q_{2}(x) \\
u_{a}=\frac{-m}{k_{0}} q_{4} \\
S=\frac{I_{h}}{k_{4}} q_{5}-\frac{I_{h}}{k_{4}} \zeta
\end{array}\right.
$$

where $b=\sqrt{T / E I}, a_{i}, i=1, \ldots, 4$, are the constants and their values are uniquely determined by the boundary conditions (25) and $\zeta$ can be computed by $\varphi^{\prime}(0), \omega(l), \omega^{\prime}(0), \omega^{\prime \prime}(0)$, and $\omega^{\prime \prime \prime}(0)$. Thus, we can obtain that $A^{-1}$ is a compact operator using Sobolev embedding theorem.

Under Assumption 1, using (9), (11), and (12), we can conclude that $F(t)$ is locally Lipschitz continuous. Thus, the closed-loop system is well posed [38]. Finally, if $Y_{0} \in H$, the closed-loop system has a unique solution, which can be expressed as

$$
Y=\Theta(t) Y_{0}+\int_{0}^{t} \Theta(t-s) F(s) \mathrm{d} s,
$$

where $\Theta(t)$ is the semigroup associated with $A$.

4.2. Uniform Boundedness. Consider the candidate Lyapunov function as

$$
E(t)=E_{1}(t)+E_{2}(t)+E_{3}(t)+E_{4}(t)
$$

where the energy term $E_{1}(t)$, auxiliary term $E_{2}(t)$, small crossing term $E_{3}(t)$, and estimation error term $E_{4}(t)$ are proposed as

$$
\left\{\begin{array}{l}
E_{1}(t)=\frac{\rho}{2} \int_{0}^{l}[\dot{w}+(r+x) \dot{\theta}]^{2} \mathrm{~d} x+\frac{T}{2} \int_{0}^{l}\left(w^{\prime}\right)^{2} \mathrm{~d} x \\
+\frac{\mathrm{EI}}{2} \int_{0}^{l}\left(w^{\prime \prime}\right)^{2} \mathrm{~d} x \\
E_{2}(t)=\frac{m}{2} u_{a}^{2}(t)+\frac{k_{3}}{2} \theta_{e}^{2}(t)+\frac{I_{h}}{2} S^{2}(t), \\
E_{3}(t)=\gamma_{1} \rho \int_{0}^{l}(r+x)\left[w^{\prime}+\theta_{e}(t)\right][\dot{w}+(r+x) \dot{\theta}(t)] \mathrm{d} x, \\
E_{4}(t)=\frac{1}{2}\left(\tilde{m}^{2}+\widetilde{T}^{2}+\widetilde{\mathrm{EI}}^{2}+{\widetilde{I_{h}}}^{2}\right)+\frac{1}{2}\left[\tilde{d}_{1}^{2}(t)+\tilde{d}_{2}^{2}(t)\right] .
\end{array}\right.
$$

Using Young's inequality for $E_{3}(t)$ yields

$$
\begin{aligned}
\left|E_{3}(t)\right|= & \gamma_{1} \rho\left|\int_{0}^{l}(r+x)\left[w^{\prime}+\theta_{e}(t)\right][\dot{w}+(r+x) \dot{\theta}] \mathrm{d} x\right| \\
\leq & \frac{\gamma_{1} \rho(r+l)}{2} \int_{0}^{l}\left(w^{\prime}\right)^{2} \mathrm{~d} x+\frac{\gamma_{1} \rho(r+l)}{\delta_{1}} \theta_{e}^{2}(t) \\
& +\gamma_{1} \rho(r+l)\left(\frac{1}{2}+\delta_{1}\right) \int_{0}^{l}[\dot{w}+(r+x) \dot{\theta}]^{2} \mathrm{~d} x \\
\leq & \lambda\left[E_{1}(t)+E_{2}(t)\right]
\end{aligned}
$$

where $\delta_{1}, \lambda=\gamma_{1}(r+l) \max \left\{\rho / T, 1+2 \delta_{1}, 2 \rho / \delta_{1} k_{3}\right\}>0$.

From (30), we can obtain

$$
-\lambda\left[E_{1}(t)+E_{2}(t)\right] \leq E_{3}(t) \leq \lambda\left[E_{1}(t)+E_{2}(t)\right] .
$$


Therefore, we further obtain

$$
\begin{aligned}
0< & \vartheta_{1}\left[E_{1}(t)+E_{2}(t)+E_{4}(t)\right] \leq E(t) \\
& \leq \vartheta_{2}\left[E_{1}(t)+E_{2}(t)+E_{4}(t)\right],
\end{aligned}
$$

where $\vartheta_{1}=1-\gamma_{1}(r+l) \max \left\{\rho / T, 1+2 \delta_{1}, 2 \rho / \delta_{1} k_{3}\right\}>0$ and $\vartheta_{1}=1-\gamma_{1}(r+l) \max \left\{\rho / T, 1+2 \delta_{1}, 2 \rho / \delta_{1} k_{3}\right\}>1$.

The following lemma is proposed as a useful tool to obtain our main results.

Lemma 3. The time derivative of the candidate Lyapunov function $E(t)$ has an upper bound as

$$
\dot{E}(t) \leq-\vartheta E(t)+\varepsilon,
$$

where $\vartheta$ and $\varepsilon$ are two positive constants.

Proof. See Appendix A.

According to the above proposed lemmas, the stability theorem of the closed-loop flexible spacecraft system can be given as follows.

Theorem 1. For a flexible spacecraft system described by (2)-(5), under the proposed adaptive boundary control scheme (7), Assumption 1, and the bounded initial conditions, it can be obtained that the closed-loop flexible spacecraft system is uniformly bounded. The elastic deflection $w(x, t)$ and tracking error $\theta_{e}(t)$ remain in the compact sets $\Omega$ and $\Omega_{e}$ given by

$$
\begin{aligned}
\Omega= & \left\{w(x, t) \in R|| w(x, t) \mid \leq \sqrt{\frac{\rangle_{1}}{\vartheta_{1}}\left[E(0)+\frac{\varepsilon}{\vartheta}\right]},\right. \\
& \forall(x, t) \in[0, l] \times[0, \infty)\}, \\
\Omega_{e}= & \left\{\theta_{e}(t) \in R|| \theta_{e}(t) \mid \leq \sqrt{\frac{\rangle_{2}}{\vartheta_{1}}\left[E(0)+\frac{\varepsilon}{\vartheta}\right]}, \quad \forall t \in[0, \infty)\right\} .
\end{aligned}
$$

Proof. See Appendix B.

\section{Simulation}

In order to demonstrate the feasibility and effectiveness of the proposed control scheme, the simulation is carried out employing the finite difference method. Let the initial conditions of the flexible spacecraft system be $w(x, 0)=0.6 x, \dot{w}(x, 0)=0, \forall x \in[0, l], \quad \theta(0)=0.4$, and $\dot{\theta}(0)=0$. The system parameters are listed as follows: $\rho=8 \mathrm{~kg} / \mathrm{m}, \quad l=10 \mathrm{~m}, \quad r=0.5 \mathrm{~m}, \quad m=5 \mathrm{~kg}, \quad \mathrm{EI}=120 \mathrm{Nm}^{2}$, $T=10 \mathrm{~N}, I_{h}=300 \mathrm{Ns} / \mathrm{m}$, and $c=0.0001 \mathrm{~kg} / \mathrm{ms}$. The external boundary disturbances are given as

$$
\left\{\begin{array}{l}
d_{1}(t)=\sin (0.1 \pi t)+\sin (0.2 \pi t), \\
d_{2}(t)=\cos (0.1 \pi t) .
\end{array}\right.
$$

The elastic deflection and attitude angle of the flexible spacecraft system without control are shown in Figures 3 and 4,

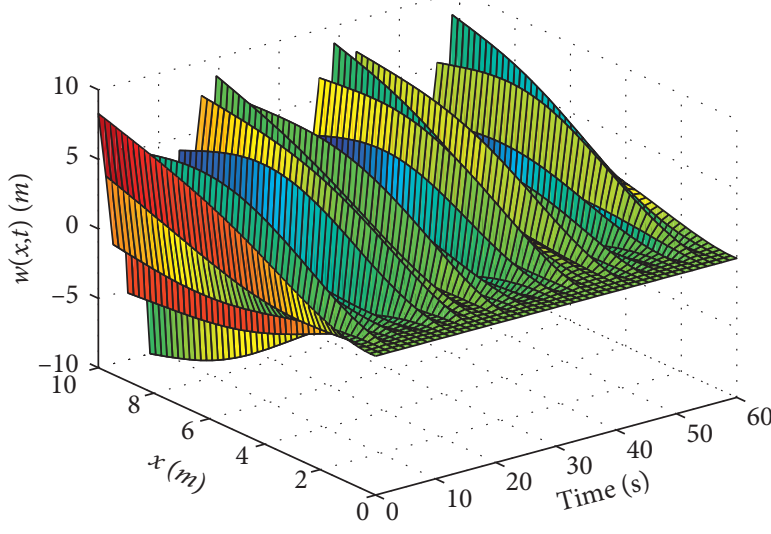

FIGURE 3: Deflection of the spacecraft without control.

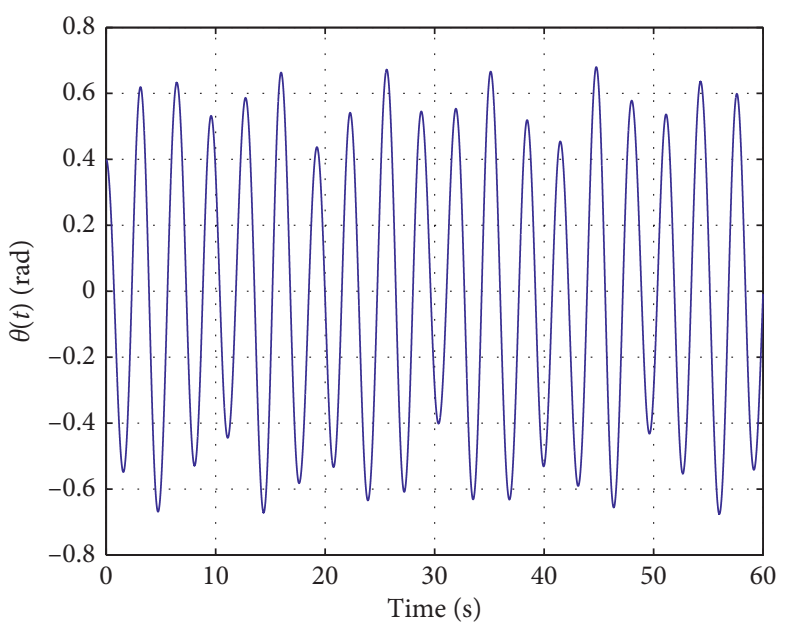

Figure 4: Attitude angle of the spacecraft without control.

respectively. Figures 5 and 6 display the deflection and attitude angle of the flexible spacecraft with the proposed adaptive boundary control scheme (7), respectively. Corresponding control inputs are described by Figure 7, where the control parameters are selected as follows: $k_{0}=10$, $k_{1}=1, k_{2}=12, k_{3}=6000$, and $k_{4}=100$. In addition, the estimates of upper bounds of boundary disturbances are shown in Figure 8. Figure 9 depicts the estimates of system parameters.

From Figures 3 and 4, it can be observed that the deflection $w(x, t)$ is quite large and the attitude angle (s) widely exceeds the desired attitude $\theta_{d}=0.2(\mathrm{rad})$. From Figures 5 and 6 , it is clear that the proposed adaptive boundary control scheme (7) can reduce effectively the deflection of flexible appendage and track the desired attitude after $20 \mathrm{~s}$. These imply that great performances of tracking attitude and vibration reduction can be obtained based on the designed control scheme. From Figure 8, the control scheme proposed in this paper can stabilize the flexible spacecraft system in spite that the estimation error values of upper bounds of boundary disturbances cannot converge completely to zero. It can conclude from Figure 9 that the estimates of system 


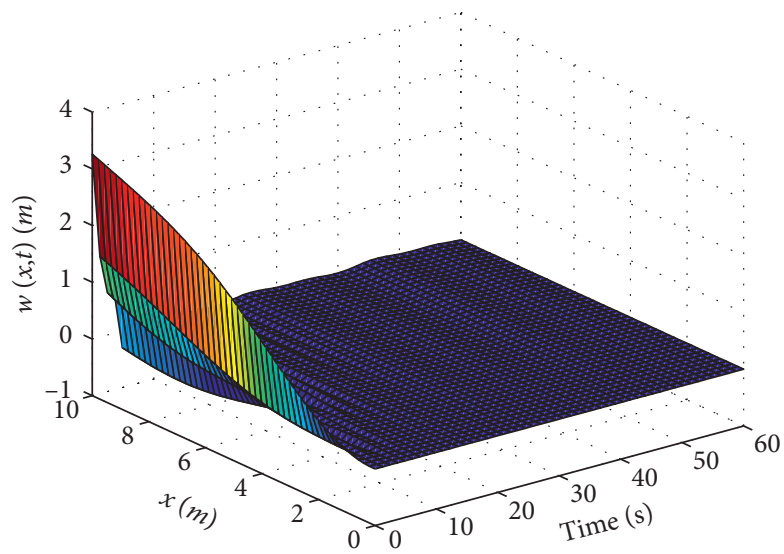

Figure 5: Deflection of the spacecraft with adaptive boundary control.

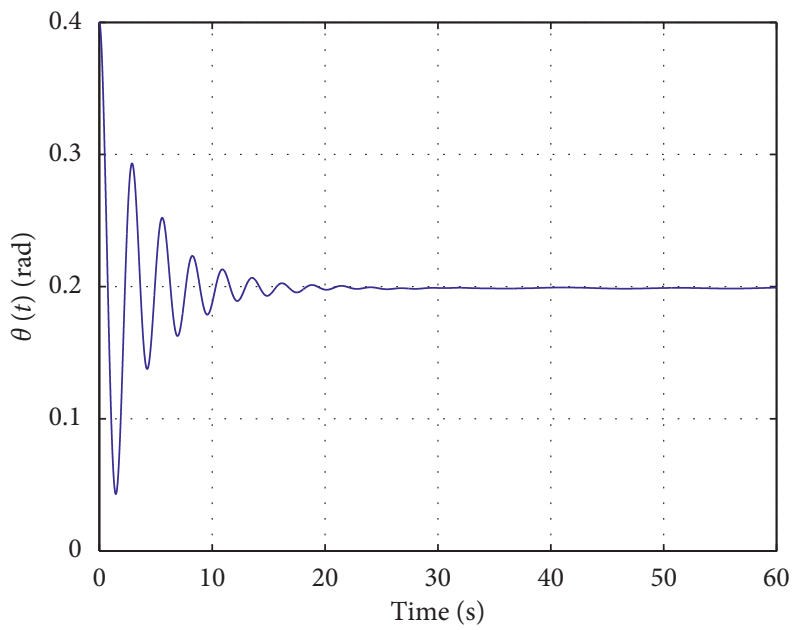

FIGURE 6: Attitude angle of the spacecraft with adaptive boundary control.


Figure 7: Control inputs.

Figure 8: Estimates of $\bar{d}_{1}$ and $\bar{d}_{2}$. 

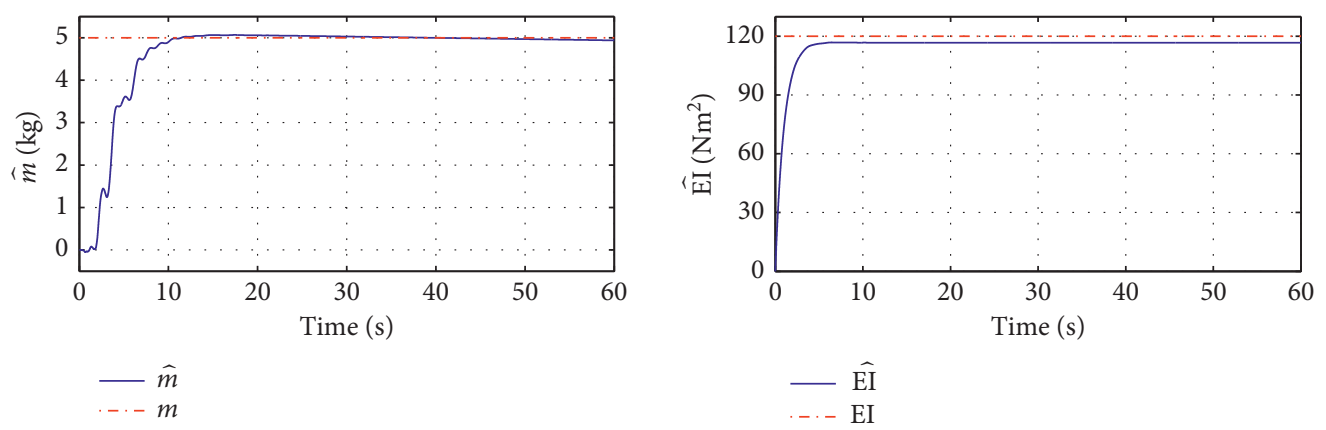

(a)
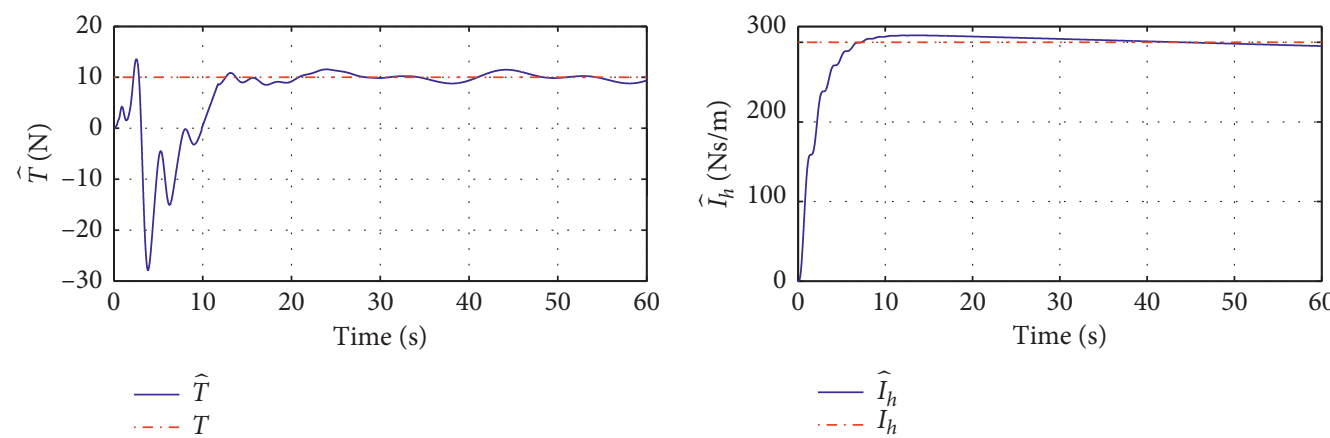

(c)

(d)

FIgURE 9: Estimates of system parameters.

parameters converge to the neighborhood of their true values.

\section{Conclusion}

In this paper, the control problems of vibration suppression and tracking attitude for a flexible spacecraft system subject to external disturbances and parameter uncertainties have been addressed. We have designed an adaptive boundary control scheme (7) to reduce the elastic deflection of flexible appendage and guarantee the convergence of the tracking error of attitude angle. Two disturbance adaptive laws (11) and (12) and adaptive laws (10) have been developed to eliminate the impacts of unknown boundary disturbances and parameter uncertainties. The well posedness and uniform boundedness of the closed-loop system have been proven. The simulation results have illustrated the effectiveness of the proposed control scheme.

It is worth mentioning that the proposed control scheme can avoid the problem of spillover instability and control all system modes since it is designed based on the original PDE model. In addition, the proposed control scheme is relatively practical control strategy since it is easy to be implemented by sensors and actuators embedded at the system boundaries. The proposed adaptive boundary scheme has great robustness for the unknown external disturbance. In future, we will investigate the other advanced control schemes such as fuzzy control and active disturbance rejection control to stabilize the flexible spacecraft system studied in this paper.

\section{Appendix}

\section{Proof of Lemma 3:}

Taking the time derivative of (28) results in

$$
\dot{E}(t)=\dot{E}_{1}(t)+\dot{E}_{2}(t)+\dot{E}_{3}(t)+\dot{E}_{4}(t) .
$$

Differentiating $E_{1}(t)$ and then substituting the governing equation (3), we have

$$
\dot{E}_{1}(t)=A_{1}+A_{2}+A_{3},
$$

where $A_{1}, A_{2}$, and $A_{3}$ are defined as

$$
\left\{\begin{array}{l}
A_{1}=T \int_{0}^{l} \dot{w}^{\prime} w^{\prime} \mathrm{d} x, \\
A_{2}=\mathrm{EI} \dot{w}^{\prime \prime} w^{\prime \prime} \mathrm{d} x, \\
A_{3}=\int_{0}^{l}[\dot{w}+(r+x) \dot{\theta}(t)]\left[T w^{\prime \prime}-\mathrm{E} \mathrm{I} w^{\prime \prime \prime \prime}\right] \mathrm{d} x \\
-c \int_{0}^{l}[\dot{w}+(r+x) \dot{\theta}(t)]^{2} \mathrm{~d} x .
\end{array}\right.
$$


Applying integration by parts for $A_{1}$ and $A_{2}$ leads to

$$
\begin{aligned}
A_{1} & =T\left[\left.\dot{w} w^{\prime}\right|_{x=0} ^{l}-\int_{0}^{l} \dot{w} w^{\prime \prime} \mathrm{d} x\right] \\
& =-T \int_{0}^{l} \dot{w} w^{\prime \prime} \mathrm{d} x+T w^{\prime}(l, t) \dot{w}(l, t), \\
A_{2} & =\mathrm{EI}\left[\left.\dot{w}^{\prime} w^{\prime \prime}\right|_{x=0} ^{l}-\int_{0}^{l} \dot{w}^{\prime} w^{\prime \prime \prime} \mathrm{d} x\right] \\
& =\mathrm{EI}\left[\left.\dot{w}^{\prime} w^{\prime \prime}\right|_{x=0} ^{l}-\left.\dot{w} w^{\prime \prime \prime}\right|_{x=0} ^{l}+\int_{0}^{l} \dot{w} w^{\prime \prime \prime \prime} \mathrm{d} x\right] \\
& =\mathrm{EI} \int_{0}^{l} \dot{w} w^{\prime \prime \prime \prime} \mathrm{d} x-\operatorname{EI} \dot{w}(l, t) w^{\prime \prime \prime}(l, t) .
\end{aligned}
$$

Substituting (A.4) and (A.5) into (A.2) yields

$$
\begin{aligned}
\dot{E}_{1}(t)= & -c \int_{0}^{l}[\dot{w}+(r+x) \dot{\theta}(t)]^{2} \mathrm{~d} x \\
& +[\dot{w}(l, t)+(r+l) \dot{\theta}(t)]\left[T w^{\prime}(l, t)-\mathrm{EI} w^{\prime \prime \prime}(l, t)\right] \\
& +\dot{\theta}(t)\left[r \mathrm{EI} w^{\prime \prime \prime}(0, t)-\operatorname{EI} w^{\prime \prime}(0, t)-T w(l, t)\right] .
\end{aligned}
$$

Differentiating $E_{2}(t)$ with respect to time $t$ and substituting (7) yields

$$
\begin{aligned}
\dot{E}_{2}(t)= & -k_{0} u_{a}^{2}(t)-k_{4} S^{2}(t)-\gamma_{1} k_{3} \theta_{e}^{2}(t)-\gamma_{1} \widetilde{I}_{h} S(t) \dot{\theta}(t) \\
& +u_{a}(t)\left[u_{d_{1}}(t)+d_{1}(t)\right]+S(t)\left[u_{d_{2}}(t)+d_{2}(t)\right] \\
& +S(t)\left[T w(l, t)+\mathrm{EI} w^{\prime \prime}(0, t)-r \mathrm{EI} w^{\prime \prime \prime}(0, t)\right] \\
& +u_{a}(t)\left[\widetilde{T} w \prime(l, t)-\widetilde{\mathrm{E}} w^{\prime \prime \prime}(l, t)-\widetilde{m}\left(k_{1} \dot{w}^{\prime}(l, t)\right.\right. \\
& \left.\left.-k_{2} \dot{w}^{\prime \prime \prime}(l, t)\right)\right] .
\end{aligned}
$$

Differentiating $E_{3}(t)$, we have

$$
\begin{aligned}
\dot{E}_{3}(t)= & \gamma_{1} \rho \int_{0}^{l}(r+x)\left[\dot{w}^{\prime}+\dot{\theta}(t)\right][\dot{w}+(r+x) \dot{\theta}(t)] \mathrm{d} x \\
& +\gamma_{1} \rho \int_{0}^{l}(r+x)\left[w^{\prime}+\theta_{e}(t)\right][\ddot{w}+(r+x) \ddot{\theta}(t)] \mathrm{d} x .
\end{aligned}
$$

Utilizing integration by parts derives

$$
\begin{aligned}
\gamma_{1} \rho \int_{0}^{l}(r+x)\left[\dot{w}^{\prime}+\dot{\theta}(t)\right][\dot{w}+(r+x) \dot{\theta}(t)] \mathrm{d} x \\
=\frac{\gamma_{1} \rho(r+l)}{2}[\dot{w}(l, t)+(r+l) \dot{\theta}(t)]^{2}-\frac{\gamma_{1} \rho r^{3}}{2} \dot{\theta}^{2}(t) \\
\quad-\frac{\gamma_{1} \rho}{2} \int_{0}^{l}[\dot{w}+(r+x) \dot{\theta}(t)]^{2} \mathrm{~d} x .
\end{aligned}
$$

Substituting the governing equation (3), we obtain

$$
\begin{aligned}
\gamma_{1} \rho \int_{0}^{l}(r+x)\left[w^{\prime}+\theta_{e}(t)\right] \\
\cdot[\ddot{w}+(r+x) \ddot{\theta}(t)] \mathrm{d} x=I_{1}+I_{2}+I_{3}+I_{4},
\end{aligned}
$$

in which

$$
\left\{\begin{array}{l}
I_{1}=\gamma_{1} T \int_{0}^{l}(r+x) w w^{\prime \prime} \mathrm{d} x, \\
I_{2}=-\gamma_{1} \mathrm{EI} \int_{0}^{l}(r+x) w w^{\prime \prime \prime} \mathrm{d} x \\
I_{3}=-\gamma_{1} c \int_{0}^{l}(r+x) w[\dot{w}+(r+x) \dot{\theta}(t)] \mathrm{d} x \\
I_{4}=\gamma_{1} \int_{0}^{l}(r+x) \theta_{e}(t)\left[T w^{\prime \prime}-\mathrm{EI} w^{\prime \prime \prime}\right] \mathrm{d} x \\
-c \gamma_{1} \theta_{e}(t) \int_{0}^{l}(r+x)[\dot{w}+(r+x) \dot{\theta}(t)] \mathrm{d} x
\end{array}\right.
$$
yield

Using integration by parts for $I_{1}$ and $I_{2}$ and applying (7)

$$
\begin{aligned}
I_{1}= & -\frac{\gamma_{1} T}{2} \int_{0}^{l}\left(w^{\prime}\right)^{2} \mathrm{~d} x+\frac{\gamma_{1} T(r+l)}{2}\left[w^{\prime}(l, t)\right]^{2}, \\
I_{2}= & -\frac{3 \gamma_{1} E I}{2} \int_{0}^{l}\left(w^{\prime \prime}\right)^{2} \mathrm{~d} x-\frac{\gamma_{1} \operatorname{EI}(r+l)}{2}\left[w^{\prime \prime}(0, t)\right]^{2} \\
& -\gamma_{1}(r+l) \mathrm{EI} w^{\prime}(l, t) w^{\prime \prime \prime}(l, t) .
\end{aligned}
$$

Apply triangle inequality and Lemma 1 for $I_{3}$ results in

$$
I_{3} \leq \frac{c \gamma_{1}(r+l)}{2}\left[l \int_{0}^{l}(w)^{\prime 2} \mathrm{~d} x+\int_{0}^{l}[\dot{w}+(r+x) \dot{\theta}(t)]^{2} \mathrm{~d} x\right]
$$

By using integration by parts and Lemma 1, we derive

$$
\begin{aligned}
I_{4} \leq & \gamma_{1} c \delta_{4} \int_{0}^{l}[\dot{w}+(r+x) \dot{\theta}(t)]^{2} \mathrm{~d} x+\gamma_{1} T(r+l) \delta_{2}\left[w^{\prime}(l, t)\right]^{2} \\
& +\gamma_{1} \theta_{e}(t)\left[r \mathrm{EI} w^{\prime \prime \prime}(0, t)-\mathrm{EI} w^{\prime \prime}(0, t)-T w(l, t)\right] \\
& +\left(\frac{\gamma_{1} T(r+l)}{\delta_{2}}+\frac{\gamma_{1} \mathrm{EI}(r+l)}{\delta_{3}}+\frac{\gamma_{1} c}{\delta_{4}}\right) \theta_{e}^{2}(t) \\
& +\gamma_{1} \operatorname{EI}(r+l) \delta_{3}\left[w^{\prime \prime \prime}(l, t)\right]^{2},
\end{aligned}
$$

where $\delta_{2}, \delta_{3}, \delta_{4}>0$.

Combining with (A.12)-(A.15), we have 


$$
\begin{aligned}
\dot{E}_{3}(t) \leq & -\left(\frac{\gamma_{1} \rho}{2}-\frac{\gamma_{1}(r+l) c}{2}-\gamma_{1} c \delta_{4}\right) \int_{0}^{l}[\dot{w}+(r+x) \dot{\theta}(t)]^{2} \mathrm{~d} x \\
& -\frac{3 \gamma_{1} \mathrm{EI}}{2} \int_{0}^{l}\left(w^{\prime \prime}\right)^{2} \mathrm{~d} x-\left(\frac{\gamma_{1} T}{2}-\frac{\gamma_{1}(r+l) c}{2}\right) \int_{0}^{l}\left(w^{\prime}\right)^{2} \mathrm{~d} x \\
& +\gamma_{1} \mathrm{EI}(r+l)\left[w^{\prime \prime \prime}(l, t)\right]^{2}+\frac{\gamma_{1} \rho(r+l)}{2} \\
& \cdot[\dot{w}(l, t)+(r+l) \dot{\theta}(t)]^{2} \\
& +\gamma_{1}(r+l)\left[T\left(1+2 \delta_{2}\right)\left[w^{\prime}(l, t)\right]^{2}\right. \\
& \left.-\mathrm{EI} w^{\prime}(l, t) w^{\prime \prime \prime}(l, t)\right] \\
& +\gamma_{1} \theta_{e}(t)\left[r \mathrm{EI} w^{\prime \prime \prime}(0, t)-\mathrm{EI} w^{\prime \prime}(0, t)-T w(l, t)\right] \\
& +\left(\frac{\gamma_{1} T(r+l)}{\delta_{2}}+\frac{\gamma_{1} \mathrm{EI}(r+l)}{\delta_{2}}+\frac{\gamma_{1} c}{\delta_{4}}\right) \theta_{e}^{2}(t) .
\end{aligned}
$$

Substituting (10) into the fourth term of (A.1) and utilizing triangle inequality lead to

$$
\begin{aligned}
\dot{E}_{4}(t) \leq & -\frac{\eta_{1}}{2} \widetilde{m}^{2}-\frac{\eta_{2}}{2} \widetilde{T}^{2}-\frac{\eta_{3}}{2} \widetilde{\mathrm{EI}}^{2}-\frac{\eta_{4}}{2} \widetilde{I}_{h}^{2}-\frac{\sigma_{1}}{2} \widetilde{d}_{1}^{2}(t)-\frac{\sigma_{2}}{2} \widetilde{d}_{2}^{2}(t) \\
& +\frac{\eta_{1}}{2} m^{2}+\frac{\eta_{2}}{2} T^{2}+\frac{\eta_{3}}{2} \mathrm{EI}^{2}+\frac{\eta_{4}}{2} I_{h}^{2}+\frac{\sigma_{1}}{2} \bar{d}_{1}^{2}+\frac{\sigma_{2}}{2}{\widetilde{d_{2}}}^{2} \\
& +\widetilde{d}_{1}(t)\left|u_{a}(t)\right|+\widetilde{d}_{2}(t)|S(t)|-\gamma_{1} \widetilde{I}_{h} S(t) \dot{\theta}(t) \\
& +u_{a}(t)\left[\widetilde{T} w^{\prime}(l, t)-\widetilde{m}\left(k_{1} \dot{w}^{\prime}(l, t)-k_{2} \dot{w}^{\prime \prime \prime}(l, t)\right)\right. \\
& \left.-\widetilde{\mathrm{EI}} w^{\prime \prime \prime}(l, t)\right] .
\end{aligned}
$$

Substituting (A.6), (A.7), (A.16), and (A.17) into (A.1), we have

$$
\begin{aligned}
\dot{E}(t) \leq & -\nu_{1} \int_{0}^{l}[\dot{w}+(r+x) \dot{\theta}(t)]^{2} \mathrm{~d} x-\gamma_{1} \nu_{2} \int_{0}^{l}\left(w^{\prime}\right)^{2} \mathrm{~d} x \\
& -\frac{3 \gamma_{1} \mathrm{EI}}{2} \int_{0}^{l}\left(w^{\prime \prime}\right)^{2} \mathrm{~d} x-\nu_{3} u_{a}^{2}(t)-k_{4} S^{2}(t)-\gamma_{1} \nu_{4} \theta_{e}^{2}(t) \\
& -v_{5}[\dot{w}(l, t)+(r+l) \dot{\theta}(t)]^{2}-v_{6}\left[w^{\prime \prime \prime}(l, t)\right]^{2} \\
& -v_{7}\left[w^{\prime}(l, t)\right]^{2}-\frac{\sigma_{1}}{2} \widetilde{d}_{1}^{2}(t)-\frac{\sigma_{2}}{2} \widetilde{d}_{2}^{2}(t)-\frac{\eta_{1}}{2} \widetilde{m}^{2} \\
& -\frac{\eta_{2}}{2} \widetilde{T}^{2}-\frac{\eta_{3}}{2} \widetilde{\mathrm{EI}}^{2}-\frac{\eta_{4}}{2} \widetilde{I}_{h}^{2}+\frac{\eta_{1}}{2} m^{2}+\frac{\eta_{2}}{2} T^{2}+\frac{\eta_{3}}{2} \mathrm{EI}^{2} \\
& +\frac{\eta_{4}}{2} \mathrm{I}_{h}^{2}+\frac{\sigma_{1}}{2}{\overline{d_{1}}}^{2}+\frac{\sigma_{2}}{2} \bar{d}_{2}^{2}+\tau_{1}+\tau_{2} \\
& \leq-\vartheta_{3}\left[E_{1}(t)+E_{2}(t)+E_{4}(t)\right]+\varepsilon
\end{aligned}
$$

where the proper parameters $\gamma_{1}, k_{i}(i=0,1,2,3,4)$, and $\delta_{j}$ $(j=1, \ldots, 6)$ are chosen to satisfy the following conditions:

$$
\left\{\begin{array}{l}
v_{1}=c+\frac{\gamma_{1} \rho}{2}-\frac{\gamma_{1}(r+l) c}{2}-\gamma_{1} c \delta_{4}>0, \\
v_{2}=T-(r+l) c>0, \\
v_{3}=k_{0}-\frac{T}{2 k_{1}}>0, \\
v_{4}=k_{3}-\frac{T(r+l)}{\delta_{2}}-\frac{\mathrm{EI}(r+l)}{\delta_{3}}-\frac{c}{\delta_{4}}>0, \\
v_{5}=\frac{T}{2 k_{1}}-\frac{\gamma_{1} \rho(r+l)}{2}-\frac{\left|k_{2} T-k_{1} \mathrm{EI}\right|}{k_{1} \delta_{5}}>0, \\
v_{6}=\frac{T k_{1}}{2}-\left|k_{2} T-\gamma_{1}(r+l) \mathrm{EI}\right| \delta_{6}-\gamma_{1}(r+l) T\left(1+2 \delta_{2}\right)>0, \\
v_{7}=\frac{T k_{2}^{2}}{2 k_{1}}-\frac{\left|k_{2} T-k_{1} \mathrm{EI}\right| \delta_{5}}{k_{1}}-\gamma_{1}(r+l) \mathrm{EI} \delta_{3} \\
\vartheta_{3}=\frac{\left.\eta_{1}, \sigma_{1}, \sigma_{2}\right\}>0,}{2} m^{2}+\frac{\eta_{2}}{2} T^{2}+\frac{\eta_{3}}{2} \mathrm{EI}+\frac{\eta_{4}}{2} \mathrm{I}_{h}^{2}+\frac{\sigma_{1}}{2} \bar{d}_{1}^{2}+\frac{\sigma_{2}}{2} \bar{d}_{2}^{2}+\tau_{1}+\tau_{2} . \\
\delta_{6}
\end{array}\right.
$$

According to (32) and (A.18), we further obtain

$$
\dot{E}(t) \leq-\vartheta E(t)+\varepsilon,
$$

where $\vartheta=\vartheta_{3} / \vartheta_{2}>0$.

\section{Proof of Theorem 1:}

From Lemma 3, multiplying both sides of (33) by $e^{9 t}$ yields

$$
\frac{\partial\left(E(t) e^{9 t}\right)}{\partial t} \leq \varepsilon e^{9 t} .
$$

Integrating the above inequality, we have

$$
E(t) \leq\left[E(0)-\frac{\varepsilon}{\vartheta}\right] e^{-\vartheta t}+\frac{\varepsilon}{\vartheta} \leq E(0) e^{-\vartheta t}+\frac{\varepsilon}{\vartheta} .
$$


$E(0)$ is bounded since the system initial conditions is bounded. Inequality (B.2) implies that $E(t)$ is bounded.

Applying Lemma 1, (29), and (32) results in

$$
\left\{\begin{array}{l}
w^{2}(x, t) \leq l \int_{l}^{0}\left(w^{\prime}\right)^{2} \mathrm{~d} x \leq \varrho_{1} E_{1}(t) \leq \frac{\varrho_{1}}{\vartheta_{1}} E(t), \\
\theta_{e}^{2}(t) \leq \varrho_{2} E_{2}(t) \leq \frac{\varrho_{2}}{\vartheta_{1}} E(t), \\
\widetilde{m}^{2}, \widetilde{\mathrm{EI}}^{2}, \widetilde{T}^{2},{\widetilde{I_{h}}}^{2} \leq 2 E_{4}(t) \leq \frac{2}{\vartheta_{1}} E(t), \\
\widetilde{d}_{1}^{2}(t), \widetilde{d}_{2}^{2}(t) \leq 2 E_{4}(t) \leq \frac{2}{\vartheta_{1}} E(t),
\end{array}\right.
$$

where $\varrho_{1}=2 l / T>0$ and $\varrho_{2}=2 / k_{3}$.

Substituting (B.2) into (B.3) leads to

$$
\left\{\begin{array}{l}
|w(x, t)| \leq \sqrt{\frac{\varrho_{1}}{\vartheta_{1}}} \sqrt{\left[E(0)-\frac{\varepsilon}{\vartheta}\right] e^{-\vartheta t}+\frac{\varepsilon}{\vartheta}} \\
\leq \sqrt{\frac{\varrho_{1}}{\vartheta_{1}}\left[E(0)+\frac{\varepsilon}{\vartheta}\right]}, \quad \forall 0 \leq x \leq l, \forall t \geq 0, \\
\left|\theta_{e}(t)\right| \leq \sqrt{\frac{\varrho_{2}}{\vartheta_{1}}} \sqrt{\left[E(0)-\frac{\varepsilon}{\vartheta}\right] e^{-9 t}+\frac{\varepsilon}{\vartheta}} \\
\leq \sqrt{\frac{\varrho_{2}}{\vartheta_{1}}\left[E(0)+\frac{\varepsilon}{\vartheta}\right]}, \quad \forall t \geq 0, \\
|\tilde{m}|,|\widetilde{E I}|,|\widetilde{T}|,\left|\widetilde{I_{h}}\right|,\left|\tilde{d}_{1}(t)\right|,\left|\tilde{d}_{2}(t)\right| \\
\leq \sqrt{\frac{2}{\vartheta_{1}}} \sqrt{\left[E(0)-\frac{\varepsilon}{\vartheta}\right] e^{-\vartheta t}+\frac{\varepsilon}{\vartheta}} \\
\leq \sqrt{\frac{2}{\vartheta_{1}}\left[E(0)+\frac{\varepsilon}{\vartheta}\right]}, \quad \forall t \geq 0 .
\end{array}\right.
$$

According to Lemma 2, we can get that the closed-loop system is uniformly bounded. The error signals $\widetilde{m}, \widetilde{\mathrm{EI}}, \widetilde{T}, \widetilde{I}_{h}$, $\widetilde{d}_{1}(t)$, and $\widetilde{d}_{2}(t)$ are uniformly bounded. $w(x, t)$ and $\theta_{e}(t)$ remain in the compact sets.

\section{Data Availability}

The data used to support the findings of this study are available from the corresponding author upon request.

\section{Conflicts of Interest}

The authors declare that they have no conflicts of interest.

\section{Acknowledgments}

This work was supported in part by the National Natural Science Foundation of China (61203060, 61673181, and 81960327), Science and Technology Planning Project of Guangdong Province under Grant (2019A050510015), and Science and Technology Department of Jiangxi Province of China (20192BAB207029).

\section{References}

[1] B. Li, Q. Hu, Y. Yu, and G. Ma, "Observer-based fault-tolerant attitude control for rigid spacecraft," IEEE Transactions on Aerospace and Electronic Systems, vol. 53, no. 5, pp. 25722582, 2017.

[2] R. Chai, A. Savvaris, A. Tsourdos, S. Chai, and Y. Xia, "Optimal tracking guidance for aeroassisted spacecraft reconnaissance mission based on receding horizon control," IEEE Transactions on Aerospace and Electronic Systems, vol. 54, no. 4, pp. 1575-1588, 2018.

[3] N. Qi, Q. Yuan, Y. Liu, M. Huo, and S. Cao, "Consensus vibration control for large flexible structures of spacecraft with modified positive position feedback control," IEEE Transactions on Control Systems Technology, vol. 27, no. 4, pp. 1712-1719, 2019.

[4] Q. Hu and G. Ma, "Variable structure control and active vibration suppression of flexible spacecraft during attitude maneuver," Aerospace Science And Technology, vol. 9, no. 4, pp. 307-317, 2005.

[5] Z. Li and P. M. Bainum, "Vibration control of flexible spacecraft integrating a momentum exchange controller and a distributed piezoelectric actuator," Journal of Sound and Vibration, vol. 177, no. 4, pp. 539-553, 1994.

[6] H. J. Gao, X. Yang, and P. Shi, "Multi-objective robust, $H_{\infty}$ control of spacecraft rendezvous," IEEE Transactions on Control Systems Technology, vol. 17, no. 4, pp. 794-802, 2009.

[7] M. J. Balas, "Active control of flexible systems," Journal of Optimization Theory and Applications, vol. 25, no. 3, pp. 415-436, 1978.

[8] W. He and S. Zhang, "Control design for nonlinear flexible wings of a robotic aircraft," IEEE Transactions on Control Systems Technology, vol. 25, no. 1, pp. 351-357, 2017.

[9] C. Ren and S. P. He, "Positiveness and observer-based finitetime control for a class of Markov jump systems with some complex environment parameters," Complexity, vol. 2018, Article ID 5365493, 13 pages, 2018.

[10] S. P. He, Q. Ai, C. C. Ren, J. Dong, and F. Liu, "Finite-time resilient controller design of a class of uncertain nonlinear systems with time-delays under asynchronous switching," IEEE Transactions on Systems, Man, and Cybernetics: Systems, vol. 49, no. 2, pp. 281-286, 2018.

[11] S. He, H. Fang, M. Zhang, F. Liu, and Z. Ding, "Adaptive optimal control for a class of nonlinear systems: the online policy iteration approach," IEEE Transactions on Neural Networks and Learning Systems, vol. 31, no. 2, pp. 549-558, 2020.

[12] Z. J. Zhao and C. K. Ahn, "Boundary output constrained control for a flexible beam system with prescribed Performance," IEEE Transactions on Systems, Man, and Cybernetics: Systems, pp. 1-9, 2019. 
[13] B. Xu and P. Zhang, "Composite learning sliding mode control of flexible-link manipulator," Complexity, vol. 2017, Article ID 9430259, 6 pages, 2017.

[14] H.-N. Wu, H.-D. Wang, and L. Guo, "Disturbance rejection fuzzy control for nonlinear parabolic PDE systems via multiple observers," IEEE Transactions on Fuzzy Systems, vol. 24, no. 6, pp. 1334-1348, 2016.

[15] M. A. Demetriou, "Adaptive and optimal synchronization control of networked positive real infinite dimensional systems with virtual leader," International Journal of Adaptive Control and Signal Processing, vol. 32, no. 10, pp. 1403-1416, 2018.

[16] Z. Liu, J. Liu, and W. He, "Robust adaptive fault tolerant control for a linear cascaded ODE-beam system," Automatica, vol. 98, pp. 42-50, 2018.

[17] T. Endo, F. Matsuno, and Y. Jia, "Boundary cooperative control by flexible Timoshenko arms," Automatica, vol. 81, pp. 377-389, 2017.

[18] L. P. Jose, M. Rakhshan, M. M. Mardani et al., "Distributed saturated control for a class of semilinear PDE systems: an SOS spproach," IEEE Transactions on Fuzzy Systems, vol. 26, no. 2, pp. 749-760, 2018.

[19] H.-N. Wu and S. Feng, "Mixed fuzzy/boundary control design for nonlinear coupled systems of ODE and boundary-disturbed uncertain beam," IEEE Transactions on Fuzzy Systems, vol. 26, no. 6, pp. 3379-3390, 2018.

[20] Z. Liu, J. Liu, and W. He, "Modeling and vibration control of a flexible aerial refueling hose with variable lengths and input constraint," Automatica, vol. 77, pp. 302-310, 2017.

[21] Z. J. Liu, X. Y. He, Z. J. Zhao, C. K. Ahn, and H. X. Li, "Vibration control for spatial aerial refueling hoses with bounded actuators," IEEE Transactions on Industrial Electronics, 2020, In press.

[22] Y. Liu, Z. Zhao, and W. He, "Boundary control of an axially moving accelerated/decelerated belt system," International Journal of Robust and Nonlinear Control, vol. 26, no. 17, pp. 3849-3866, 2016.

[23] W. He, S. Zhang, and S. S. Ge, "Adaptive control of a flexible crane system with the boundary output constraint," IEEE Transactions on Industrial Electronics, vol. 61, no. 8, pp. 4126-4133, 2014.

[24] Z. J. Zhao, X. Y. He, Z. Ren, and G. Wen, "Boundary adaptive robust control of a flexible riser system with input nonlinearities," IEEE Transactions on Systems, Man, and Cybernetics: Systems, vol. 49, no. 10, pp. 1971-1980, 2018.

[25] Z. J. Zhao, X. Y. He, Z. Ren, and G. Wen, "Output feedback stabilization for an axially moving system," IEEE Transactions on Systems, Man, and Cybernetics: Systems, vol. 49, no. 12, pp. 2374-2383, 2018.

[26] X. Tong, X. Zhao, and S. Zhao, "Load reduction of a monopile wind turbine tower using optimal tuned mass dampers," International Journal of Control, vol. 90, no. 7, pp. 1283-1298, 2017.

[27] W. He, T. T. Meng, X. Y. He et al., "Iterative learning control for a flapping wing micro aerial vehicle under distributed disturbances," IEEE Transactions on Cybernetics, vol. 49, no. 4, pp. 1524-1535, 2018.

[28] Z. J. Zhao, X. Y. He, and C. K. Ahn, "Boundary disturbance observer-based control of a vibrating single-link flexible manipulator," IEEE Transactions on Systems, Man, and Cybernetics: Systems, 2019, In press.

[29] Z. Zhao, C. K. Ahn, and H.-X. Li, "Boundary antidisturbance control of a spatially nonlinear flexible string system," IEEE
Transactions on Industrial Electronics, vol. 67, no. 6, pp. 4846-4856, 2020.

[30] L. J. Yang, Z. J. Liu, and S. Zhang, "Single parameter adaptive control of unknown nonlinear systems with tracking error constraints," Complexity, vol. 2018, Article ID 6457354, 19 pages, 2018.

[31] W. He, X. Y. He, and S. S. Ge, "Vibration control of flexible marine riser systems with input saturation," IEEE/ASME Transactions on Mechatronics, vol. 21, no. 1, pp. 254-265, 2016.

[32] Y. Liu, F. Guo, X. Y. He, and Q. Hui, "Boundary control for an axially oving system with input restriction based on disturbance observers," IEEE Transactions on Systems, Man, and Cybernetics: Systems, vol. 49, no. 11, pp. 2242-2253, 2018.

[33] W. Kang and B.-Z. Guo, "Stabilisation of unstable cascaded heat partial differential equation system subject to boundary disturbance," IET Control Theory \& Applications, vol. 10, no. 9, pp. 1027-1039, 2016.

[34] Z. J. Zhao, C. K. Ahn, and X. H. Li, "Dead zone compensation and adaptive vibration control of uncertain spatial flexible riser systems," IEEE/ASME Transactions on Mechatronics, 2020, In press.

[35] F.-F. Jin and B.-Z. Guo, "Lyapunov approach to output feedback stabilization for the Euler-Bernoulli beam equation with boundary input disturbance," Automatica, vol. 52, pp. 95-102, 2015.

[36] C. D. Rahn, Mechatronic Control of Distributed Noise and Vibration, Springer, New York, NY, USA, 2001.

[37] W. He, Y. Dong, and C. Sun, "Adaptive neural impedance control of a robotic manipulator with input saturation," IEEE Transactions on Systems, Man, and Cybernetics: Systems, vol. 46, no. 3, pp. 334-344, 2016.

[38] A. Pazy, Semigroups of Linear Operators and Applications to Partial Differential Equations, Springer-Verlag, New York, NY, USA, 1983. 